\title{
Ultrasensitive Isotope Trace Analyses with a Magneto-Optical Trap
}

\author{
C. Y. Chen, ${ }^{1}$ Y. M. Li, ${ }^{1}$ K. Bailey, ${ }^{1}$ T. P. O'Connor, ${ }^{1}$ L. Young, ${ }^{2}$ \\ Z.-T. Lu ${ }^{1 *}$
}

\begin{abstract}
Laser manipulation of neutral atoms has been used to count individual krypton85 and krypton-81 atoms present in a natural krypton gas sample with isotopic abundances in the range of $10^{-11}$ and $10^{-13}$, respectively. This method of isotope trace analysis is free of contamination from other isotopes and elements and can be applied to several different isotope tracers for a wide range of applications. The demonstrated detection efficiency is $1 \times 10^{-7}$. System improvements could increase the efficiency by many orders of magnitude.
\end{abstract}

Ultrasensitive isotope trace analysis has been an important tool in modern science. Two well-developed methods, low-level counting (LLC) (1) and accelerator mass spectrometry (AMS) (2), have been used for dating as well as for studying the transport processes in the ocean, atmosphere, and groundwater $(3,4)$. They also have applications in studying cosmic rays (5), detecting solar neutrinos (6), and searching for exotic particles (7). Here we report the development of a widely applicable method, atom trap trace analysis (ATTA), which can enhance the capability and expand the applications of ultrasensitive isotope trace analyses.

As a test we focused on the long-lived ${ }^{85} \mathrm{Kr}$ and ${ }^{81} \mathrm{Kr}$ isotopes. ${ }^{85} \mathrm{Kr}$ has a half-life of 10.8 years and an abundance of $\sim 10^{-11}$ (3) and ${ }^{81} \mathrm{Kr}$ has a half-life of $2.3 \times 10^{5}$ years and an abundance of $(5.9 \pm 0.6) \times 10^{-13}(8)$. The present-day ${ }^{85} \mathrm{Kr}$ in the environment has been released primarily by nuclear-fuel reprocessing plants. Its abundance has increased a millionfold since the 1950 s. It has been used as a general tracer to study air and ocean currents (3), date shallow groundwater (3), and monitor nuclear-fuel reprocessing activities (9). In contrast, ${ }^{81} \mathrm{Kr}$ is produced in the upper atmosphere by cosmic ray-induced reactions and is shielded from manmade fission products by stable ${ }^{81} \mathrm{Br} .{ }^{81} \mathrm{Kr}$ is an ideal tracer for dating ancient groundwater and ice on the time scale of $10^{5}$ to $10^{6}$ years $(10)$, but accurate measurement has been difficult (11). A method of counting ${ }^{81} \mathrm{Kr}$ atoms would also make possible a solar neutrino detector that is sensitive to both ${ }^{7} \mathrm{Be}$ and ${ }^{8} \mathrm{~B}$ neutrinos from the sun $(6,12)$.

LLC generally has been used to measure the abundance of an isotope by counting its

${ }^{1}$ Physics Division, and ${ }^{2}$ Chemistry Division, Argonne National Laboratory, 9700 South Cass Avenue, Argonne, IL 60439, USA.

*To whom correspondence should be addressed. Email: lu@anl.gov nuclear decays. It is currently used to count ${ }^{85} \mathrm{Kr}$. Although once used to count ${ }^{81} \mathrm{Kr}(8)$, this is no longer possible because of the high present-day decay background of ${ }^{85} \mathrm{Kr}$. Taking a different approach, AMS counts atoms instead of decays, thereby greatly enhancing the detection efficiency and avoiding the radioactive background problem. AMS is now routinely used as the standard method of ${ }^{14} \mathrm{C}$ dating (4). Recently, AMS has been applied to count ${ }^{81} \mathrm{Kr}$ and date groundwater (13). In this work, a high-energy cyclotron (K1200, MSU) was used to remove all the electrons from the atoms so that ${ }^{81} \mathrm{Kr}$ can be separated from its abundant isobar ${ }^{81} \mathrm{Br}$. Laser-based techniques, such as resonance ionization spectrometry (RIS) (14) and photon burst mass spectrometry (15), have the potential of being simple and efficient. Using RIS, Hurst and co-workers have counted ${ }^{81} \mathrm{Kr}$ atoms in an enriched sample with over $50 \%$ efficiency (16). So far, neither laser-based technique has demonstrated the isotopic selectivity required to count ${ }^{81} \mathrm{Kr}$ or ${ }^{85} \mathrm{Kr}$ in a natural sample.

The ATTA method we developed is based on laser manipulation of neutral atoms. ATTA differs significantly from previous methods in that it is free of contamination from different isotopes or elements. Therefore, ATTA can tolerate impure gas samples, does not require a special operation environment, and is sensitive to certain proposed anomalously heavy isotopes (7).

Our design is based on a type of magnetooptical trap (MOT) system that had been used to trap various metastable noble gas atoms (17). Trapping krypton atoms in the $5 \mathrm{~s}[3 / 2]_{2}$ metastable level (lifetime $=40 \mathrm{~s}$ ) is accomplished by exciting the $5 \mathrm{~s}[3 / 2]_{2}-5 \mathrm{p}[5 / 2]_{3}$ transition with laser light with a wavelength of $811 \mathrm{~nm}$ generated by a Ti-sapphire ring laser. The laser frequency, after shifting by acousto-optical modulators (AOM) to account for the isotope shift, is locked to a reference absorption line of the abundant
${ }^{83} \mathrm{Kr}$ atoms in a cell. Two repump sidebands are generated via additional AOMs to optically pump the atoms into the $5 \mathrm{~s}[3 / 2]_{2}$ level $\left(F=13 / 2\right.$ for ${ }^{85} \mathrm{Kr}, F=11 / 2$ for ${ }^{81} \mathrm{Kr}$ ) where they can be excited by the trapping light (Fig. 1). In the analysis, a krypton gas sample is injected into the system through a $0.1-\mathrm{mm}$ diameter nozzle, around which a dc discharge is maintained. A fraction (about $1 \times 10^{-4}$ ) of the atoms are excited into the $5 \mathrm{~s}[3 / 2]_{2}$ level by the discharge and, after exiting the discharge region, remain in this metastable level until they hit walls. Two-dimensional transverse cooling is used to reduce the atomic beam divergence and amplify the atom flux in the forward direction by a factor of 20 . The thermal $\left(300^{\circ} \mathrm{C}\right)$ atoms are then decelerated by the Zeeman slowing technique (18) and loaded into a MOT (19). Atoms remain trapped for an average of $1.8 \mathrm{~s}$ when the vacuum is maintained at $2 \times 10^{-8}$ torr. This trap system can capture the abundant ${ }^{83} \mathrm{Kr}$ (isotopic abundance $=11.5 \%$ ) atoms at a rate of $2 \times 10^{8} \mathrm{~s}^{-1}$. The ratio of the capture rate to the injection rate gives a total capture efficiency of $1 \times 10^{-7}$.

With expected capture rates between $10^{-3}$ and $10^{-2} \mathrm{~s}^{-1}$ for the rare krypton isotopes, the system must be capable of detecting a single atom in the trap (20). In the trap, a single atom scatters resonant photons at a rate of $10^{7} \mathrm{~s}^{-1}$, of which $1 \%$ are collected, spatially filtered to reduce background light, and then focused onto an avalanche photodiode (EG\&G, SPCM-AQ-212) with a specified photon counting efficiency of $25 \%$. For single-atom detection, each trapping laser beam is set at $1 \mathrm{~cm}$ diameter and $2 \mathrm{~mW} / \mathrm{cm}^{2}$, and the magnetic field gradient of the MOT is set at $16 \mathrm{G} / \mathrm{cm}$ along its axis. Under these conditions, fluorescence from a single atom induces a signal of 15 kilocounts per second



Fig. 1. Optimum laser frequencies for trapping ${ }^{85} \mathrm{Kr}$ and ${ }^{81} \mathrm{Kr}$ atoms. $f_{0}$ is the frequency of the reference transition in ${ }^{83} \mathrm{Kr} . f_{85}$ and $f_{81}$ are frequencies of light for trapping ${ }^{85} \mathrm{Kr}$ and ${ }^{81} \mathrm{Kr}$ respectively. Note only hyperfine levels relevant to trapping are drawn. 
Table 1. Some isotopes that might be analyzed with AT TA. This list does not intend to include all possible cases but rather a few examples. $\mathrm{Ca}, \mathrm{Sr}, \mathrm{Cs}$, and $\mathrm{Pb}$ can be trapped in their ground levels.

\begin{tabular}{|c|c|c|c|}
\hline Isotope & Applications & Trap transition & Reference \\
\hline${ }^{39} \mathrm{Ar}$ & Trace deep ocean currents & $4 \mathrm{~s}[3 / 2]_{2} \rightarrow 4 \mathrm{p}[5 / 2]_{3}, 812 \mathrm{~nm}$ & (3) \\
\hline${ }^{41} \mathrm{Ca}$ & $\begin{array}{l}\text { Date ancient bones; also a } \\
\text { medical tracer }\end{array}$ & $4{ }^{1} \mathrm{~S}_{\mathrm{o}} \rightarrow 4{ }^{1} \mathrm{p}^{\circ}{ }_{1}, 423 \mathrm{~nm}$ & (27) \\
\hline${ }^{90} \mathrm{Sr}$ & Monitor nuclear fallout & $5{ }^{1} \mathrm{~S}_{\mathrm{O}} \rightarrow 5^{1} \mathrm{p}^{\circ}{ }_{1}, 461 \mathrm{~nm}$ & (28) \\
\hline${ }^{135,137} \mathrm{Cs}$ & Monitor long-lived nuclear waste & $6^{2} \mathrm{~S}_{1 / 2} \rightarrow 6^{2} \mathrm{P}_{3 / 2}, 852 \mathrm{~nm}$ & (29) \\
\hline${ }^{205} \mathrm{~Pb}$ & $\begin{array}{l}\text { Measure time-integrated solar } \\
\text { neutrino flux }\end{array}$ & $6{ }^{3} \mathrm{P}_{\mathrm{o}} \rightarrow 7{ }^{3} \mathrm{p}^{\circ}{ }_{1}, 283 \mathrm{~nm}$ & (6) \\
\hline
\end{tabular}

(kcps) and the background is $18 \mathrm{kcps}$ (Fig. 2). These laser and field parameters differ significantly from those needed for an optimum trap loading rate $(3-\mathrm{cm}$ diameter, $10 \mathrm{~mW} /$ $\mathrm{cm}^{2}$, and $8-\mathrm{G} / \mathrm{cm}$ field gradient). The trap loading conditions generate too much background light to permit single atom detection so we switch at $2 \mathrm{~Hz}$ between the optimal parameters for loading and detection, with each phase lasting $0.25 \mathrm{~s}$.

We have trapped and counted ${ }^{85} \mathrm{Kr}$ and ${ }^{81} \mathrm{Kr}$ atoms from natural krypton gas (Fig. 2). The frequency settings of the trapping laser and the two sidebands (Fig. 1) are in good agreement with previous spectroscopic measurements obtained with enriched ${ }^{85} \mathrm{Kr}$ gas and enriched ${ }^{81} \mathrm{Kr}$ gas (21). The fluorescence from a trapped ${ }^{85} \mathrm{Kr}$ atom decreases as the laser frequency is tuned away from resonance, following the expected Lorentzian shape (Fig. 3). We also mapped the atom capture rate versus laser frequency (Fig. 4B) and showed that the maximum loading was achieved with the frequency detuned about 4 $\mathrm{MHz}$ below resonance, consistent with the results observed on ${ }^{83} \mathrm{Kr}$ traps (Fig. 4A). Furthermore, we performed repeated tests under conditions in which a ${ }^{85} \mathrm{Kr}$ trap should not work, such as turning off repump sidebands and tuning the laser frequency above resonance, and these tests always yielded zero atom counts. When we required an atom fluorescence signal to be $>10 \mathrm{kcps}$, atom counts were observed in a frequency band only 12

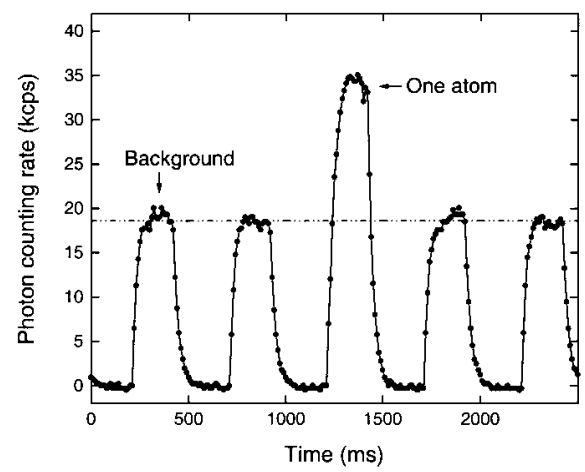

Fig. 2. Signal of a single trapped ${ }^{85} \mathrm{Kr}$ atom. During loading time, the photon-count rate was low because the counter was blocked for protection from overexposure.
MHz wide (Fig. 4C). This test shows that the recorded counts are due to laser-trapped ${ }^{85} \mathrm{Kr}$ atoms and that no background atom counts from other isotopes or elements have been observed. We conducted similar tests with ${ }^{81} \mathrm{Kr}$. We alternated the laser frequency settings between the optimum trap condition (Fig. 1) and, as a background check, $5 \mathrm{MHz}$ above the optimum condition where trapping does not work. In this case we counted 12 ${ }^{81} \mathrm{Kr}$ atoms in a total of 5 hours at the optimum trap condition, and we obtained 0 counts in another total of 5 hours during background checks.

Previous efforts to develop a laser-based technique have encountered serious problems with contamination from nearby abundant isotopes. ATTA is immune from isotope contamination for several reasons: fluorescence is collected in only a small region $(0.5 \mathrm{~mm}$ diameter) around the trap center; a trapped

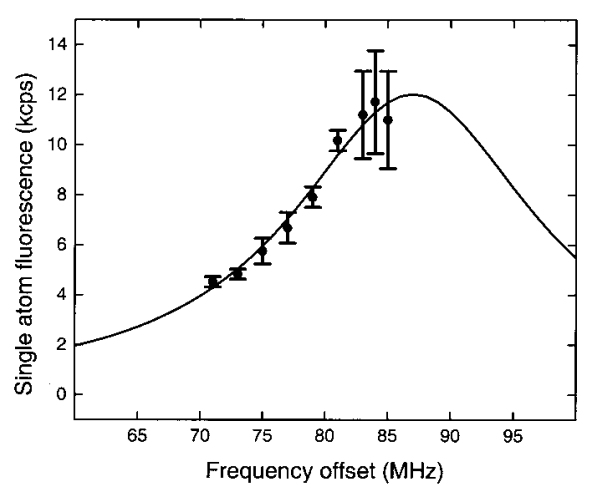

Fig. 3. Single-atom fluorescence versus laser frequency offset. Laser frequency offset indicates detuning from the reference line. Measured photon-count rates are fitted to a Lorentzian function, rates $\propto\left[\left(f-f_{r}\right)^{2}+(\gamma / 2)^{2}\right]^{-1}$. Here, $f_{r}$ is the resonant transition frequency of ${ }^{85} \mathrm{Kr}, f$ is the laser frequency, and $\gamma=$ $\Gamma(1+s)^{1 / 2}$ is the power-broadened linewidth. We fixed $f_{\mathrm{r}}=87 \mathrm{MHz}$ and $\Gamma=5.3 \mathrm{MHz}$ according to previous spectroscopy measurements (21). The resulting saturation parameter $s=19 \pm 3$. Error bars represent change in fluorescence between different atoms. We attribute the cause of large fluctuations near resonance to the fact that the atom trajectory partially extends beyond the viewing region as the trap temperature increases near resonance. This effect was also observed on ${ }^{83} \mathrm{Kr}$ traps with a charge-coupled device camera. atom is cooled to a speed below $1 \mathrm{~m} / \mathrm{s}$ so that its laser-induced fluorescence is virtually Doppler-free (19); a trapped atom allows a long observation time, during which $10^{3}$ scattered signal photons are collected; and trapping allows the temporal separation of capture and detection so that both capture efficiency and detection sensitivity can be optimized. In the case of counting ${ }^{85} \mathrm{Kr}$, photons scattered off of the walls and the metastable $\mathrm{Kr}$ atomic beam (22) induce a background of $18 \mathrm{kcps}$ on the photon counter. With a 200ms observation period, the signal of a single trapped atom is 40 times the noise $(1 \sigma)$ of the background photons. Our design also provides additional features, although they were unnecessary in the case of krypton, to further improve isotopic selectivity, such as chopping off the atomic beam before detecting the trapped atom or sending in a laser beam to selectively push out (or de-excite) the atoms of contaminant isotopes.

Besides isotopic selectivity, another important characteristic is detection efficiency. The efficiency of our system depends on the discharge current, laser power, and optical alignment. At one particular setting, we measured capture rates of ${ }^{83} \mathrm{Kr},{ }^{85} \mathrm{Kr}$, and ${ }^{81} \mathrm{Kr}$, which were $(1.5 \pm 0.3) \times 10^{8} \mathrm{~s}^{-1},(1.9 \pm$ $0.3) \times 10^{-2} \mathrm{~s}^{-1}$, and $(1.3 \pm 0.4) \times 10^{-3}$ $\mathrm{s}^{-1}$, respectively (23). If we assume the same detection efficiency for all three isotopes, then we get isotopic abundances of $(1.5 \pm$

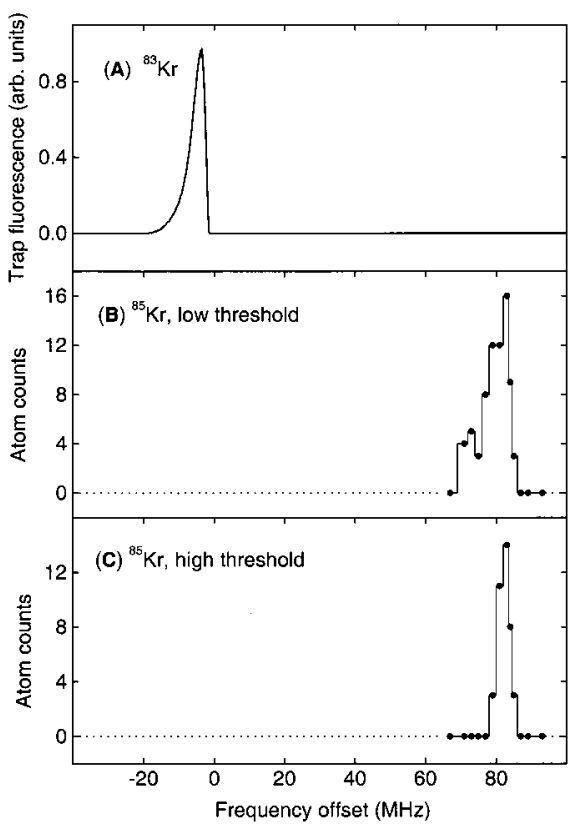

Fig. 4. (A) Fluorescence of trapped ${ }^{83} \mathrm{Kr}$ atoms versus laser frequency. Fluorescence was measured with a low-gain photodiode detector. (B and C) Number of ${ }^{85} \mathrm{Kr}$ atoms counted versus laser frequency. Each data point represents number of atoms counted in $20 \mathrm{~min}$, with 10 min each for loading and counting. Fluorescence threshold, over which an atom is counted, was set at $3 \mathrm{kcps}(\mathrm{B})$ and $10 \mathrm{kcps}(\mathrm{C})$. 
$0.4) \times 10^{-11}$ for ${ }^{85} \mathrm{Kr}$ and $(1.0 \pm 0.4) \times$ $10^{-12}$ for ${ }^{81} \mathrm{Kr}$, which are in good agreement with previous measurements using other methods $(3,8,13)$. The capture efficiencies can be calibrated with enriched samples of known isotopic abundance to correct for any isotope-dependent effects and to measure isotopic ratios in unknown samples.

Our system has achieved an efficiency of $10^{-7}$. Use of this system to measure the abundance of ${ }^{85} \mathrm{Kr}$ to within $10 \%$ would require 2 hours and a krypton sample of 3 $\mathrm{ml}$, whereas measurement of ${ }^{81} \mathrm{Kr}$ to within $10 \%$ would require 2 days and a sample of $60 \mathrm{ml}$. This limits the current system to atmospheric applications in which large samples of gas are readily available. It is possible to raise the efficiency many orders of magnitude through improvements such as cryogenic cooling in the discharge region (24) and recirculation of krypton gas (25). Other proposed schemes (26) with an ultraviolet (UV) laser instead of a dc discharge to excite the atoms to the metastable level via a two-photon transition could dramatically improve detection efficiency.

ATTA can be applied to many different isotopes (Table 1). Laser trapping is well established on alkali, alkali earth, and noble gas elements. Trapping other elements is generally more difficult because of both the complexity of their ground-level structures and the lack of suitable UV lasers. Some of these problems may be overcome with future advances in UV laser technology.

\section{References and Notes}

1. J. R. Arnold and W. F. Libby, Science 113, 111 (1951).

2. R. A. Muller, Science 196, 489 (1977); D. E. Nelson et al., Science 198, 507 (1977); C. L. Bennett et al., Science 198, 508 (1977).

3. International Atomic Energy Agency, Isotope of Noble Gases as Tracers in Environmental Studies (International Atomic Energy Agency, Vienna, Austria, 1992).

4. Proc. Sixth International Conference on AMS, Nucl. Instr. Meth. 123 (1997).

5. S. Baumgartner et al., Science 279, 1330 (1998)

6. R. Davis Jr., A. K. Mann, L. Wolfenstein, Annu. Rev. Nucl. Part. Sci. 39, 467 (1989); J. N. Bahcall and R. K. Ulrich, Rev. Mod. Phys. 60, 297 (1988).

7. R. A. Muller, L. W. Alvarez, W. R. Holley, E. J. Stephenson, Science 196, 521 (1977); R. N. Cahn and S. L. Glashow, Science 213, 607 (1981).

8. H. H. Loosli and H. Oeschger, Earth Planet. Sci. Lett. 7, 67 (1969).

9. F. von Hippel, D. H. Albright, B. G. Levi, Sci. Am. 253, 40 (Sept., 1985)

10. H. Oeschger, Nucl. Instrum. Methods Phys. Res. B 29, 196 (1987).

11. J. R. Petit et al., Nature 399, 429 (1999); C. Lorius et al., Nature 316, 591 (1985)

12. G. S. Hurst, M. G. Payne, S. D. Kramer, C. H. Chen, Phys. Today 33, 24 (Sept. 1980)

13. P. Collon et al., Nucl. Instr. Meth. B123, 122 (1997); P. Collon, thesis (University of Vienna, Austria, 1999).

14. V. S. Letokhov, Laser Photoionization Spectroscopy (Academic Press, Orlando, 1987); G. S. Hurst and M. G. Payne, Principles and Applications of Resonance Ionisation Spectroscopy (Adam Hilger, Bristol, UK, 1988).

15. R. D. LaBelle, C. S. Hansen, M. M. Mankowski, W. M. Fairbank Jr., Phys. Rev. A 54, 4461 (1996); B. D. Cannon and T. J. Whitaker, Appl. Phys. B 38, 57 (1985).
16. S. D. Kramer et al., Nucl. Instrum. Methods Phys. Res. B 17, 395 (1986)

17. M. Walhout, H. J. L. Megens, A. Witte, S. L. Rolston, Phys. Rev. A 48, R879 (1993); F. Shimizu, K. Shimizu, H. Takuma, Chem. Phys. 145, 327 (1990).

18. W. Phillips and H. Metcalf, Phys. Rev. Lett. 48, 596 (1982); T. E. Thomas, S. W. Dapore-Schwartz, M. D. Ray, G. P. Lafayatis, Phys. Rev. Lett. 67, 3483 (1991).

19. E. L. Raab, M. Prentiss, A. E. Cable, S. Chu, D. E. Pritchard, Phys. Rev. Lett. 59, 2631 (1987).

20. Z. Hu and H. J. Kimble, Opt. Lett. 19, 1888 (1994); D. Haubrich et al., Europhys. Lett. 34, 663 (1996).

21. B. D. Cannon, Phys. Rev. A 47, 1148 (1993).

22. Metastable atoms quench upon colliding with walls; therefore, they do not exist in background as a vapor. In other applications when ground-level atoms are used, cryopanels can be installed to reduce the density of background atoms.

23. Rate calculations are based on loading time only, which is one-half of real time.

24. W. Roojakkers, W. Hogervorst, W. Vassen, Opt. Commun. B 123, 321, (1996)
25. B. E. Lehman, D. F. Rauber, N. Thonnard, R. D. Willis, Nucl. Instrum. Methods Phys. Res. B 28, 571 (1987).

26. G. S. Hurst, M. G. Payne, R. C. Phillips, J. W. T. Dabbs, B. E. Lehmann, J. Appl. Phys. 55, 1278 (1984).

27. W. Henning et al., Science 236, 725 (1987).

28. K. Zimmer et al., Appl. Phys. B 59, 117 (1994).

29. J.-H. Chao and C.-L. Tseng, Nucl. Instrum. Methods Phys. Res. A 372, 275 (1996).

30. Supported by the U.S. Department of Energy, Nuclear Physics Division. L.Y. was supported by the Office of Basic Energy Sciences, Division of Chemical Sciences (contract W-31-109-ENG-38). We thank C. Wieman and W. Kutschera for stimulating discussions. In particular, Z.-T.L. had private communications with C. Wieman about Wieman's earlier proposal to count ${ }^{90} \mathrm{Sr}$ atoms with a MOT. We also thank D. Lin for his contribution to the earlier phase of this project, M. Walhout for showing us discharge designs, and $\mathrm{B}$. Cannon for calculating the hyperfine structure of ${ }^{81} \mathrm{Kr}$.

\title{
Similarity of the $C$. elegans Developmental Timing Protein LIN-42 to Circadian Rhythm Proteins
}

\author{
Mili Jeon, ${ }^{1 *}$ Heather F. Gardner, ${ }^{2 *}$ Eric A. Miller, ${ }^{2} \dagger$ \\ Jodie Deshler, ${ }^{2}+$ Ann E. Rougvie ${ }^{1,2} \S$
}

\begin{abstract}
The Caenorhabditis elegans heterochronic genes control the relative timing and sequence of many events during postembryonic development, including the terminal differentiation of the lateral hypodermis, which occurs during the final (fourth) molt. Inactivation of the heterochronic gene lin-42 causes hypodermal terminal differentiation to occur precociously, during the third molt. LIN-42 most closely resembles the Period family of proteins from Drosophila and other organisms, proteins that function in another type of biological timing mechanism: the timing of circadian rhythms. Per mRNA levels oscillate with an approximately 24-hour periodicity. lin-42 mRNA levels also oscillate, but with a faster rhythm; the oscillation occurs relative to the approximately 6-hour molting cycles of postembryonic development.
\end{abstract}

The timing of distinct biological processes within a cell or organism is carefully controlled. One class of temporal regulators, exemplified by the $C$. elegans heterochronic genes, times the onset of developmental events. These genes control the relative timing of diverse stage-specific events during postembryonic development such as dauer larva formation, vulva formation, and the terminal differentiation of the hypodermis (1). In other organisms, members of this general

${ }^{1}$ Department of Biochemistry, Molecular Biology, and Biophysics; 'Department of Genetics, Cell Biology, and Development, University of Minnesota, St. Paul, MN 55108, USA.

*These authors contributed equally to this work $\uparrow$ Present address: Department of Epidemiology, University of North Carolina, Chapel Hill, NC 27599, USA. †Present address: Department of Biology, University of Wisconsin, River Falls, WI 54022, USA.

$\S$ To whom correspondence should be addressed. heterochronic gene class include the Teopod genes and Glossy15 of maize, hasty of Arabidopsis, and anachronism of Drosophila (2). Mutations in these genes either advance or retard expression of certain developmental programs.

A second class of temporal regulators comprises the "clock" components that regulate circadian rhythms, the approximately 24hour periodicity of biological processes such as sleep-wake cycles in humans (3). Genes that control circadian rhythms have been cloned from several organisms, including Drosophila, Neurospora, and mouse. Although shared motifs have been observed between certain circadian rhythm proteins, shared motifs between these proteins and the timing factors that control developmental progression have not been reported previously. Here we demonstrate that the C. elegans heterochronic gene $\operatorname{lin}-42$ encodes a protein 